\title{
Les oreillons chez les jeunes adultes : le canari dans la mine de charbon
}

$\mathrm{P}$ our beaucoup de gens, les oreillons rappellent une époque révolue de maladies infantiles que la vaccination permet maintenant d'éviter. Or, les oreillons ont refait surface et des éclosions récentes dans beaucoup de pays industrialisés ont touché surtout des adolescents et des jeunes adultes (de I5 à 26 ans) dont beaucoup sont étudiants. Au Canada, une éclosion a fait son apparition plus tôt cette année en Nouvelle-Écosse (voir page I37). On y a signalé plus de 370 cas et le nombre continue d'augmenter. L'éclosion s'est propagée aux autres provinces de l'Atlantique, en Ontario, en Alberta et en Colombie-Britannique. On a signalé aussi des éclosions au Royaume-Uni (> 50000 en 2004-2005), aux ÉtatsUnis ( $>2500$ en 2006) et en Espagne (> I300 en 2006-2007), de même que plus tôt en Alberta ( $>$ I8o en 2002) et en NouvelleÉcosse ( $>30$ en $2005^{-2006^{1}}$ ). Dans la plupart des cas, le malade avait reçu au moins une dose de vaccin rougeole-oreillonsrubéole (ROR) pendant l'enfance et, au cours de l'éclosion survenue aux États-Unis en 2006, 46 \% en avaient reçu deux doses. Les souches d'oreillons à paramyxovirus aux États-Unis et en Nouvelle-Écosse ressemblent au génotype G du RoyaumeUni, qui s'est propagé à grande échelle.

Comme il n'y a pas d'agent antiviral efficace pour la prévention ou le traitement des oreillons, le seul recours, c'est la prévention par la vaccination et le contrôle de la propagation par l'isolement au cours de la période infectieuse. Il est toutefois difficile sur le plan logistique de circonscrire l'éclosion au moyen d'un «filet» de vaccins de rappel étant donné la diversité de la population des adolescents et des jeunes adultes. De même, l'isolement présente bien des difficultés, si l'on en juge par l'expérience récente de la NouvelleÉcosse : beaucoup de jeunes adultes ont pu être exposés aux oreillons pendant qu'ils célébraient la Saint-Patrick dans des bars bondés. Essayer de leur dire de s'auto-isoler lorsqu'ils n'ont ressenti aucun symptôme, c'est un coup d'épée dans l'eau. Il y a une autre raison pour laquelle les jeunes adultes constituent une population idéale pour les éclosions d'oreillons : ils ont tendance à partager des logements exigus et ont plus de contacts rapprochés avec des gens du même âge que les adultes plus âgés ou les enfants.

Aussi, beaucoup de jeunes adultes ont reçu une seule dose de vaccin ROR, ce qui alourdit le risque posé par les oreillons dans ce groupe d'âge. Même si une seule dose de vaccin est efficace à $80 \%$, il n'en reste pas moins que $20 \%$ des sujets immunisés demeurent vulnérables. Même après deux doses, $5 \%$ des sujets peuvent ne pas avoir encore acquis une immunité. De plus, une vaccination antérieure peut causer davantage d'infections infracliniques, ce qui augmente la possibilité de propagation. Les jeunes adultes qui ont contracté les oreillons mais qui se sentent bien ne consulteront sans doute pas le médecin et, même s'ils le font, les médecins qui ne connaissent pas bien cette maladie risquent de rater le diagnostic. Or, les conséquences peuvent être graves. Jusqu'à $38 \%$ des hommes qui contractent les oreillons après la puberté auront une orchite et environ I3 \% auront une baisse de fécondité. En outre, la méningo-encéphalite atteint environ 250 cas sur 100 ooo et entraîne un taux de mortalité d'environ $2 \%{ }^{2}$.

\section{Que faire?}

Les travailleurs de la santé doivent être plus vigilants et repérer rapidement les cas d'oreillons afin que l'on puisse contenir efficacement la maladie. Il doit aussi y avoir un moyen de diffuser rapidement, à la fois aux praticiens des soins de santé et au public, de l'information sur les risques, les symptômes et la nécessité de l'isolement (voir www.gov.ns.ca/hpp/mumps /health-professionals.html et www.gov.ns.ca/hpp/mumps/ assessments.html).

On pourrait de plus réduire la taille de la population à risque chez les adolescents et les jeunes adultes au Canada en administrant une deuxième dose de vaccin ROR. De multiples stratégies sont possibles à cet égard : implanter un programme provincial de rappel pour ceux et celles qui ne sont plus à l'école, exiger deux doses de vaccin ROR avant l'inscription au collège ou à l'université et élaborer des façons créatrices de lancer des programmes de rappel chez les adolescents qui ne fréquentent pas l'école et chez les jeunes adultes au travail ou dans les endroits où ces groupes se rencontrent. Étant donné la taille et la complexité de ces initiatives nécessaires de rappel, un financement fédéral est essentiel.

L'éclosion actuelle d'oreillons au Canada a démontré une fois de plus le besoin d'un registre national des vaccinations afin que les provinces et les territoires puissent savoir rapidement qui est à risque et qui ne l'est pas.

Apprendre à contrôler ces éclosions d'oreillons plus efficacement offre une importante occasion d'étudier des méthodes d'éducation rapide, des procédures novatrices de notification des contacts et d'isolement, ainsi que des mesures créatrices de vaccination rapide chez une population mobile de jeunes à risque. Ces éclosions d'oreillons sont un peu comme le fameux canari dans la mine de charbon : elles nous préviennent des lacunes de notre approche actuelle du contrôle des maladies infectieuses. Par le passé, la population des adolescents et des jeunes adultes a été touchée à grande échelle par des maladies graves et par la propagation d'autres maladies virales pandémiques, et en particulier la grippe. Nous avons beaucoup à apprendre sur la façon de contrôler la propagation d'infections virales respiratoires sérieuses dans cette population. Ces éclosions d'oreillons nous offrent une bonne occasion de le faire.

\section{Noni MacDonald MD}

Rédactrice de la section Santé publique, JAMC

Ken Flegel MDCM

Rédacteur associé principal, JAMC

Pour l'équipe de rédaction de l'éditorial (Paul C. Hébert, Matthew Stanbrook, Barbara Sibbald et Amir Attaran)

\section{REFERENCES}

I. Watson-Creed G, Saunders A, Scott J, et al. Two successive outbreaks of mumps in Nova Scotia among vaccinated adolescents and young adults. JAMC 2006;175:483-8. 2. Caplan CE. Mumps in the era of vaccines. JAMC I999;160:865-6. 